Olivares, P.R.; Hernandez-Mocholi, M.; Merellano-Navarro, E.; Gusi, N.; Collado-Mateo, D. (2019). Analysis of Age on Fitness Reliability in Elderly. Revista Internacional de Medicina y Ciencias de la Actividad Física y el Deporte vol. 19 (76) pp. 627-639 Http://cdeporte.rediris.es/revista/revista76/artanalisis1088.htm

DOI: $10.15366 /$ rimcafd2019.76.005

\title{
ORIGINAL
}

\section{ANÁLISIS DE LA EDAD SOBRE LA FIABILIDAD DE PRUEBAS FITNESS EN MAYORES}

\section{ANALYSIS OF AGE ON FITNESS RELIABILITY IN ELDERLY}

\author{
Olivares, P.R. ${ }^{1}$; Hernandez-Mocholi, M. ${ }^{2}$; Merellano-Navarro, E. ${ }^{3}$; Gusi, N. ${ }^{4}$ y \\ Collado-Mateo, D. ${ }^{5}$
}

1 PhD, Facultad de Educación, Psicología y Ciencias del Deporte, Universidad de Huelva, Huelva (España). Instituto de Actividad Física y Salud. Universidad Autónoma de Chile (Chile) olivares.pedro@gmail.com

2 MSc, Facultad de Ciencias del Deporte, Universidad de Extremadura (España) mihemo04@gmail.com

$3 \mathrm{PhD}$, Instituto de Actividad Física y Salud. Universidad Autónoma de Chile (Chile) emerellanon@uautonoma.cl

${ }^{4}$ PhD, Facultad de Ciencias del Deporte, Universidad de Extremadura (España) ngusi@unex.es

5 PhD, Facultad de Ciencias del Deporte, Universidad de Extremadura (España) Instituto de Actividad Física y Salud. Universidad Autónoma de Chile (Chile) danicolladom@gmail.com

Financiación

El estudio fue apoyado por el Fondo Interno de Investigación de la Universidad Autónoma de Chile (DIUA115-2017). El autor MAHM contó con el apoyo de la Beca del Ministerio de Educación, Cultura y Deporte de España (FPU3839). El autor DCM cuenta con el apoyo de la Beca del Ministerio de Educación, Cultura y Deporte de España (FPU14 / 01283).

Código UNESCO / UNESCO Code: 320107 Geriatría / Geriatrics Clasificación Consejo de Europa / Council of Europe Classification: 11 Medicina del Deporte / Sport Medicine

Recibido 16 de enero de 2018 Received January 16, 2018

Aceptado 27 de noviembre de 2018 Accepted November 27, 2018

\section{RESUMEN}

El presente trabajo tiene como objetivo analizar la fiabilidad de varias pruebas de condición física en adultos mayores en función de la edad. Los participantes fueron 135 mujeres mayores entre 60 y 90 años y se distribuyó en 5 grupos de edad. Todos los participantes realizaron una batería de pruebas de condición 
física con un intervalo entre mediciones de 1 semana. Los índices de fiabilidad obtenidos en las pruebas dinamometría bi-manual y 6 minutos caminando fueron excelentes ICC> 0,90, mientras que en el resto fueron buenos (ICC 0,70-0,89). No se encontraron diferencias estadísticamente significativas en el error de medida de estas pruebas entre grupos de edad. Se concluye que la fiabilidad de las pruebas de condición física utilizadas en población adulto mayor no varía significativamente en función de la edad.

PALABRAS CLAVES: Ancianos, capacidad funcional, fiabilidad.

\section{ABSTRACT}

The aim of this study was to analyze if age affects the reliability of some fitness test widely used in elderly adults. Participants were 135 elderly women aged between 60 and 90 years old distributed into 5 age groups. All participants performed twice a battery of fitness tests with an interval between measurements of 1 week. The reliability indexes obtained in the bi-handgrip tests and 6 minutes walking were excellent (ICC>0.90), while the rest were good (ICC 0.70-0.89). No statistically significant differences were found in the measurement error of these tests between age groups. It is concluded that age does not significantly affect to the reliability of the analyzed fitness tests.

KEYWORDS: Elderly, functional capacity, reliability.

\section{INTRODUCCIÓN}

Diversas investigaciones han relacionado la condición física con la capacidad funcional (Merellano-Navarro, Collado-Mateo, García-Rubio, Gusi, \& Olivares, 2017; Rikli \& Jones, 2013; Sardinha, Santos, Marques, \& Mota, 2015) y con la calidad de vida relacionada con la salud (Chung, Zhao, Liu, \& Quach, 2017; Olivares, Gusi, Prieto, \& Hernandez-Mocholi, 2011), y se ha utilizado para medir los efectos de intervenciones y terapias físicas en personas mayores (Chang, Wang, Chen, \& Hu, 2017; Chen et al., 2017; Gusi, Hernandez-Mocholi, \& Olivares, 2015). La evaluación de la condición física puede tener un papel importante en la promoción de la actividad física y salud (Oja, 1995) debido a que a través de ella, se puede identificar a las personas que poseen riesgo de contraer enfermedades crónicas, reducir la fragilidad física y aumentar la movilidad (Chen, Lin, \& Yu, 2009).

Antes de usar cualquier prueba de condición física como herramienta de medición de los efectos de una terapia, se debe saber si las puntuaciones obtenidas son confiables y cuál es el error de medición que se debe suponer. Para analizarlo, se han publicado diversos estudios de fiabilidad específicos de las pruebas de condición física en adultos mayores (Rodriguez, Valenzuela, Gusi, Nacher, \& Gallardo, 1998; Shaulis, Golding, \& Tandy, 1994). Sin embargo, el rendimiento físico disminuye con la edad (Chen et al., 2009; Krause et al., 2009; Rikli \& Jones, 1999b) principalmente debido al deterioro de la resistencia 
aeróbica, flexibilidad, fuerza, velocidad, agilidad y equilibrio (Milanović et al., 2013) y esta disminución en el rendimiento físico podría afectar sus índices de fiabilidad. Hasta donde sabemos, no hay ningún estudio que analice el efecto de la edad en los índices de fiabilidad de las pruebas de condición física más utilizadas. El objetivo de este estudio fue analizar la fiabilidad de una batería de condición física en personas mayores según grupos de edad, lo que permite detectar con mayor precisión los cambios reales en las terapias físicas en esta población. Además, esta información permitirá una mejor interpretación de los valores normativos de estas pruebas de condición física considerando el error específico de medición en cada grupo de edad.

\section{MÉTODO}

\section{PARTICIPANTES}

Se seleccionaron 3 centros públicos de personas mayores a los cuales se les informó sobre los objetivos del estudio a través de posters y charlas informativas. Después del primer acercamiento, 116 mujeres mayores entre 60 y 90 años se interesaron en participar. Todos los participantes cumplieron con los criterios de inclusión, los cuales fueron: residir en comunidad, ser funcionalmente independiente y no poseer condiciones médicas o limitaciones físicas o cognitivas que les impidieran seguir instrucciones. Los participantes se distribuyeron en 5 grupos de edad (60-64, 65-69, 70-74, 75-79 y 80 o más). El equipo de investigación presento el protocolo del estudio a los participantes y se les solicito firmar el consentimiento informado como requisito de participación. Todos los protocolos se ajustaron a las actualizaciones de la Declaración de Helsinki. El estudio fue aprobado por el Comité de Ética Biomédica de la Universidad de Extremadura (Ref.: №028-15).

\section{EVALUACIÓN DE LA CONDICIÓN FÍSICA}

Se realizó un calentamiento de 5 minutos previo a las mediciones, el cual consistió en activación neuromuscular (movilidad articular) y ejercicios de estiramiento. El tiempo total que tomaron las mediciones fue de aproximadamente 45 minutos, y se realizó durante la mañana, entre las 9:00 a.m. y la 1:00 p.m.

Las pruebas utilizadas para evaluar la condición física fueron:

Fuerza muscular: La fuerza se midió mediante dinamometría manual (Rodríguez et al., 1998). Ambas manos se midieron utilizando un dinamómetro de mano (modelo TKK 5401, Tokio, Japón) y se consideró el valor de la suma de ambas manos como resultado.

Flexibilidad de extremidades inferiores y superiores: se midió la flexibilidad de las extremidades inferiores a través de la prueba Chair Sit and Reach (Jones, Rikli, Max, \& Noffal, 1998). Esta prueba mide la distancia entre el dedo medio y la punta del pie mientras se mantiene posición sentada. Para evaluar la 
flexibilidad de las extremidades superiores (hombros) se utilizó la prueba Back Scratch (Rikli \& Jones, 1999a). Esta consiste en una combinación de abducción de hombro, aducción y rotación interna y externa, y mide la distancia entre (o superposición) de los dedos medios detrás de la espalda. La distancia entre los dedos se puntúa como un valor negativo, y se superponen como un valor positivo. Para los efectos de medición de la flexibilidad en ambas pruebas, se midieron dos veces la izquierda y la derecha y se calculó el promedio de ambas.

Agilidad: se evaluó usando la prueba timed up-and-go (Podsiadlo, 1991). Esta prueba implica levantarse de una silla, caminar 3 metros hacia y alrededor de un cono, y regresar a la silla en el menor tiempo posible.

Equilibrio: la prueba de functional reach test se utilizó para medir el equilibrio (Duncan, Weiner, Chandler, \& Studenski, 1990). En esta prueba, se determina la distancia máxima que uno puede alcanzar más allá de la longitud de los brazos mientras se mantiene sin mover los pies.

Resistencia aeróbica: la prueba de caminata de 6 minutos se aplicó para medir la resistencia aeróbica. Esta prueba implica determinar la distancia máxima en metros que se puede caminar en 6 minutos (Rikli \& Jones, 2001).

Además, se midieron tres pruebas para la descripción de la composición corporal. Se midió las circunferencias de cintura-cadera, estatura y peso para calcular el índice de masa corporal $\left(\mathrm{IMC}, \mathrm{kg} / \mathrm{m}^{2}\right)$ y la relación cintura-cadera (RCC), respectivamente. Se calculó el porcentaje de grasa corporal (\% BF) usando un analizador de impedancia portátil, de acuerdo con las instrucciones del fabricante (Omron BF306, Omron Healthcare Europe BV, Hoofddorp, The Netherlands) (Deurenberg et al., 2001).

\section{PROCEDIMIENTO}

Los participantes fueron evaluados en dos sesiones de medición separadas por una semana de diferencia por tres evaluadores graduados de Ciencias del Deporte y con experiencia previa en la evaluación de la condición física en adultos mayores. Los evaluadores recibieron un manual de prueba desarrollado por los directores del proyecto que describe todos los procedimientos y protocolos de cada una de las pruebas, y completaron 3 sesiones de cuatro horas cada una para estandarizar los métodos de evaluación y reducir el error interno y externo.

En la primera sesión, además de la evaluación de la condición física, se aplicó un cuestionario general para recoger datos demográficos que incluyen la edad, el estado civil, el nivel educativo y el nivel de actividad física a la semana. En términos de seguridad, todos los participantes fueron evaluados usando el Cuestionario de Preparación de Actividades Físicas (PAR-Q) (Thomas, Reading, \& Shephard, 1992), y se controló la presión arterial en reposo para descartar a aquellos con enfermedad cardíaca o hipertensión no controlada. Aquellos que 
respondieron "sí" a cualquier pregunta sobre el PAR-Q o que tenían una presión arterial superior a $160 / 100 \mathrm{mmHg}$ fueron excluidos del estudio.

Todas las evaluaciones se llevaron a cabo en centros de adulto mayor que cuentan con un área interior amplia, como una sala multiuso o un gimnasio. Los participantes fueron instruidos para vestir ropa y calzado apropiado, comer una comida liviana aproximadamente 1 hora antes de las pruebas, evitar tomar bebidas alcohólicas dentro de las 24 horas anteriores y no realizar actividad física vigorosa el día antes de la evaluación.

Cada participante fue evaluado de forma individual. En primer lugar, se midió el peso, la altura, la circunferencia de cintura y cadera y el porcentaje de grasa corporal. Después, los participantes hicieron ejercicios de calentamiento general antes de comenzar las pruebas y recibieron las mismas instrucciones sobre los procedimientos de cada una de las pruebas: hacerlo lo mejor que pudieran, pero nunca presionarse hasta un punto de esfuerzo excesivo o más allá de lo que pensaban que era seguro para ellos. Los participantes completaron uno o dos ensayos para familiarizarse con los procedimientos de cada una de las pruebas, excepto con la prueba de 6 minutos caminando. Para minimizar los efectos de la fatiga, las pruebas se administraron en la siguiente secuencia: Equilibrio (functional reach), Flexibilidad (chair sit-and-reach and back scratch), Fuerza (handgrip) y Agilidad (time up and go). Después de 5 minutos de descanso, se realizó la prueba de 6-min-caminando en un circuito de 20 metros.

\section{ANÁLISIS ESTADÍSTICO}

Se utilizaron la media $( \pm S D$ La fiabilidad relativa se determinó mediante el cálculo del Coeficiente de Correlación Intraclase $\left(\mathrm{CCl}_{2,2}\right)$ y su $95 \%$ de intervalo de confianza entre los dos días de medición (Shrout \& Fleiss, 1979).

La fiabilidad absoluta se determinó mediante el cálculo de los índices Error Estándar de Medida (SEM) [SEM= DT $\sqrt{ }(1-\mathrm{CCI})$ donde DT es la desviación típica del día 1 y día 2] y el Mínimo Cambio Real (SRD) [SRD= 1.96 x $\sqrt{2}$ xSEM] (Weir, 2005). Tanto el SEM como el SRD se calcularon en términos absolutos y en porcentaje para facilitar su interpretación.

Todos los análisis se realizaron por separado según grupos de edad: 60-64, 65$69,70-74,75-79$ y 80 o más. Para analizar las diferencias entre los distintos grupos de edad se realizó un ANOVA con prueba post-hoc de Games-Howel. Para analizar la magnitud de las diferencias en el error de medida obtenido entre los distintos grupos de edad se realizó un análisis del tamaño del efecto (ES). En general, una relación de ES de 0,80 o mayor se considera grande; un ES de alrededor de 0,50 se considera moderado; y una ES de 0,20 o menos se considera pequeña (Tomas \& Nelson, 2005). 


\section{RESULTADOS}

Las características sociodemográficas de los participantes se describen en la Tabla 1. El $43.1 \%$ de los participantes no recibieron educación. El porcentaje de mujeres que viven solas aumenta en los grupos de edad superior, lo que está en consonancia con la proporción cada vez mayor de mujeres viudas, separadas o divorciadas. Con respecto al nivel de actividad física, la mayoría de las mujeres mayores indicaron que realizan más de 3 horas por semana.

\begin{tabular}{|c|c|c|c|c|c|c|}
\hline & $\begin{array}{l}\text { Todas } \\
\mathrm{n}(\%)\end{array}$ & $\begin{array}{l}60-64 \\
\mathrm{n}(\%)\end{array}$ & $\begin{array}{l}65-69 \\
\mathrm{n}(\%)\end{array}$ & $\begin{array}{l}70-74 \\
n(\%)\end{array}$ & $\begin{array}{l}75-79 \\
n(\%)\end{array}$ & $\begin{array}{l}\geq 80 \\
n(\%)\end{array}$ \\
\hline \multicolumn{7}{|l|}{ Estado civil } \\
\hline Soltera & $4(3,45)$ & $3(12,5)$ & $0(0)$ & $1(3,3)$ & $0(0)$ & $0(0)$ \\
\hline Casada & $74(63,79)$ & $12(50)$ & $18(66,6)$ & $23(76,7)$ & $15(62,5)$ & $6(42,9)$ \\
\hline Viuda / Separada / Divorciada & $38(32,76)$ & $6(37,5)$ & $9(33,3)$ & $6(20)$ & $9(37,5)$ & $8(57,1)$ \\
\hline \multicolumn{7}{|l|}{ Educación } \\
\hline Sin Educación & $50(43,10)$ & $9(42,9)$ & $11(40,7)$ & $14(46,7)$ & $7(30,4)$ & $9(64,3)$ \\
\hline Primaria & $66(56,90)$ & $12(57,1)$ & $16(59,3)$ & $16(53,3)$ & $17(69,6)$ & $5(35,7)$ \\
\hline Secundaria & $0(0)$ & $0(0)$ & $0(0)$ & $0(0)$ & $0(0)$ & $0(0)$ \\
\hline Universidad & $0(0)$ & $0(0)$ & $0(0)$ & $0(0)$ & $0(0)$ & $0(0)$ \\
\hline \multicolumn{7}{|l|}{ Vive con... } \\
\hline Sola & $34(29,31)$ & $5(23,8)$ & $7(25,9)$ & $7(23,3)$ & $8(33,3)$ & $7(50)$ \\
\hline Con mi compañero & $74(63,79)$ & $12(57,1)$ & $18(66,7)$ & $23(76,7)$ & $15(62,5)$ & $6(42,9)$ \\
\hline $\begin{array}{r}\text { Con la familia } \\
\text { (hermano/hermana, hijo/hija) }\end{array}$ & $8(6,90)$ & $4(19,0)$ & $2(7,4)$ & $0(0)$ & $1(4,2)$ & $1(7,1)$ \\
\hline \multicolumn{7}{|l|}{ Actividad física por semana } \\
\hline 0 horas/semana & $12(10,34)$ & $5(23,8)$ & $2(7,4)$ & $2(6,7)$ & $0(0)$ & $3(21,4)$ \\
\hline$<3$ horas/semana & $13(11,21)$ & $3(14,3)$ & $2(7,4)$ & $5(16,79)$ & $1(4,2)$ & $2(14,3)$ \\
\hline$\geq 3$ horas/semana & $91(78,45)$ & $13(61,9)$ & $23(85,2)$ & $23(76,7)$ & $23(95,8)$ & $9(64,3)$ \\
\hline$(\mathrm{N}=116)$ & & & & & & \\
\hline
\end{tabular}

La Tabla 2 muestra el ICC, el error estándar de medida y los valores de SRD obtenidos en ambas pruebas. En las pruebas de dinamometría bi-manual y 6 minutos caminando, la fiabilidad fue excelente (ICC> 0,90), mientras que en el resto de pruebas fue buena (ICC 0,70-0,89). El grupo de edad con menor confiabilidad fue de 75-79 y el grupo con la mejor fue de 60-64 años. 
Rev.int.med.cienc.act.fís.deporte - vol. 19 - número 76 - ISSN: 1577-0354

Tabla 2. Fiabilidad de las pruebas de condición física (test-retest) en función de grupo de edad $(n=116)$

\begin{tabular}{|c|c|c|c|c|c|c|c|c|}
\hline $\begin{array}{l}\text { Grupo de } \\
\text { edad }\end{array}$ & Prueba & $\begin{array}{l}\text { Media } 1 \\
( \pm \mathrm{DT}) \\
\end{array}$ & $\begin{array}{c}\text { Media } 2 \\
( \pm \mathrm{DT}) \\
\end{array}$ & $\mathrm{CCI}(95 \% \mathrm{IC})$ & SEM & $\begin{array}{c}\% S E \\
M\end{array}$ & SRD & $\begin{array}{c}\text { \%SR } \\
\mathrm{D}\end{array}$ \\
\hline \multicolumn{9}{|l|}{ Todos $(n=116)$} \\
\hline & $\begin{array}{l}\text { Bi-manual handgrip } \\
(\mathrm{Kg})\end{array}$ & $41,2 \pm 7,9$ & $41,3 \pm 8,3$ & $\begin{array}{c}0.93(0.90 \text { to } \\
0,95)\end{array}$ & 2,2 & 5.2 & 6.0 & 14.6 \\
\hline & $\begin{array}{l}\text { Chair Seat-and-reach } \\
(\mathrm{cm})\end{array}$ & $7.1 \pm 10,6$ & $7 \pm 10,5$ & $\begin{array}{c}0,88(0,83 \text { to } \\
0,92)\end{array}$ & 3,0 & 30,3 & 8,4 & 84,0 \\
\hline & Functional reach $(\mathrm{cm})$ & $28,7 \pm 6,8$ & $29,5 \pm 6,4$ & $\begin{array}{c}0,82(0,76 \text { to } \\
0,88\end{array}$ & 3.2 & 10.9 & 8.8 & 30.2 \\
\hline & Timed up-and-go (s) & $6,9 \pm 1,3$ & $6,9 \pm 1,3$ & $\begin{array}{c}0,87(0,82 \text { to } \\
0,91)\end{array}$ & 0,5 & 6,8 & 1,3 & 18,8 \\
\hline & 6-minute walking $(\mathrm{m})$ & $454,1 \pm 79,8$ & $455,7 \pm 77,8$ & $\begin{array}{c}0,95(0,93 \text { to } \\
0,96)\end{array}$ & 17,8 & 3,9 & 49,4 & 10,8 \\
\hline \multicolumn{9}{|l|}{$60-64(n=21)$} \\
\hline & & $\begin{array}{l}\text { Media } 1 \\
( \pm \mathrm{DT})\end{array}$ & $\begin{array}{l}\text { Media } 2 \\
( \pm \mathrm{DT})\end{array}$ & $\mathrm{CCl}(95 \% \mathrm{IC})$ & SEM & $\begin{array}{c}\% \text { SE } \\
M\end{array}$ & SRD & $\begin{array}{c}\text { \%SR } \\
\mathrm{D}\end{array}$ \\
\hline & $\begin{array}{l}\text { Bi-manual handgrip } \\
\qquad(\mathrm{Kg})\end{array}$ & $45,2 \pm 7,4$ & $45,2 \pm 8,2$ & $\begin{array}{c}0,89(0,72 \text { to } \\
0,95)\end{array}$ & 2.64 & 5.84 & 7.32 & 16.20 \\
\hline & $\begin{array}{l}\text { Chair Seat-and-reach } \\
\qquad(\mathrm{cm})\end{array}$ & $7,5 \pm 10,4$ & $6,6 \pm 11,7$ & $\begin{array}{c}0,93(0,83 \text { to } \\
0,97)\end{array}$ & 2,44 & 25,80 & 6,77 & 71,52 \\
\hline & Functional reach $(\mathrm{cm})$ & $29,5 \pm 5,8$ & $30,1 \pm 6,3$ & $\begin{array}{c}0,75(0,46 \text { to } \\
0,89)\end{array}$ & 3,56 & 11,94 & 9,88 & 33,11 \\
\hline & Timed up-and-go (s) & $6,3 \pm 1,3$ & $6,4 \pm 1,1$ & $\begin{array}{c}0,92(0,81 \text { to } \\
0,97)\end{array}$ & 0,35 & 5,53 & 0,97 & 15,33 \\
\hline & 6-minute walking $(\mathrm{m})$ & $476,9 \pm 66,8$ & $480,2 \pm 69,4$ & $\begin{array}{c}0,95(0,89 \text { to } \\
0,98)\end{array}$ & $\begin{array}{c}14,9 \\
3\end{array}$ & 3,12 & $\begin{array}{c}41,3 \\
7\end{array}$ & 8,64 \\
\hline \multicolumn{9}{|l|}{$65-69(n=27)$} \\
\hline & & $\begin{array}{l}\text { Media } 1 \\
( \pm \mathrm{DT})\end{array}$ & $\begin{array}{c}\text { Media } 2 \\
( \pm \mathrm{DT})\end{array}$ & $\mathrm{CCI}(95 \% \mathrm{IC})$ & SEM & $\begin{array}{c}\% \text { SE } \\
M\end{array}$ & SRD & $\begin{array}{c}\text { \%SR } \\
\text { D }\end{array}$ \\
\hline & $\begin{array}{l}\text { Bi-manual handgrip } \\
\qquad(\mathrm{Kg})\end{array}$ & $40,3 \pm 4.5$ & $40,6 \pm 7,7$ & $\begin{array}{c}0,92(0,82 \text { to } \\
0,96)\end{array}$ & 1.77 & 4.38 & 4.91 & 12.14 \\
\hline & $\begin{array}{l}\text { Chair Seat-and-reach } \\
(\mathrm{cm})\end{array}$ & $6,6 \pm 12,7$ & $8,1 \pm 10,5$ & $\begin{array}{c}0,87(0,73 \text { to } \\
0,93)\end{array}$ & 2,94 & 25,18 & 8,14 & 69,80 \\
\hline & Functional reach $(\mathrm{cm})$ & $29,1 \pm 4,2$ & $30,4 \pm 4,7$ & $\begin{array}{c}0,71(0,46 \text { to } \\
0,86)\end{array}$ & 2,39 & 8,03 & 6,62 & 22,25 \\
\hline & Timed up-and-go (s) & $6,8 \pm 1,3$ & $6,7 \pm 1,2$ & $\begin{array}{c}0,85(0,70 \text { to } \\
0,93)\end{array}$ & 0,47 & 7,01 & 1,31 & 19,43 \\
\hline & 6-minute walking $(\mathrm{m})$ & $463,9 \pm 83,6$ & $461,5 \pm 74,7$ & $\begin{array}{c}0,93(0,86 \text { to } \\
0,97)\end{array}$ & $\begin{array}{c}20,6 \\
4\end{array}$ & 4,46 & $\begin{array}{c}57,2 \\
1\end{array}$ & 12,36 \\
\hline \multicolumn{9}{|l|}{$70-74(\mathrm{n}=30)$} \\
\hline & & $\begin{array}{l}\text { Media } 1 \\
( \pm \mathrm{DT})\end{array}$ & $\begin{array}{c}\text { Media } 2 \\
( \pm \mathrm{DT})\end{array}$ & $\mathrm{CCl}(95 \% \mathrm{IC})$ & SEM & $\begin{array}{c}\text { \%SE } \\
M\end{array}$ & SRD & $\begin{array}{c}\text { \%SR } \\
\text { D }\end{array}$ \\
\hline & $\begin{array}{l}\text { Bi-manual handgrip } \\
\qquad(\mathrm{Kg})\end{array}$ & $41,5 \pm 6,8$ & $42,2 \pm 7,6$ & $\begin{array}{c}0,95(0,89 \text { to } \\
0,98)\end{array}$ & 1.66 & 3.96 & 4.59 & 10.97 \\
\hline & $\begin{array}{l}\text { Chair Seat-and-reach } \\
(\mathrm{cm})\end{array}$ & $11,4 \pm 8.8$ & $10,8 \pm 9,3$ & $\begin{array}{c}0,86(0,72 \text { to } \\
0,93)\end{array}$ & 3.97 & 34.64 & $\begin{array}{c}11,0 \\
1\end{array}$ & 96.00 \\
\hline & Functional reach $(\mathrm{cm})$ & $29,5 \pm 7,2$ & $30,5 \pm 6.6$ & $\begin{array}{c}0,87(0,73 \text { to } \\
0,94)\end{array}$ & 3,23 & 10,76 & 8,95 & 29,84 \\
\hline & Timed up-and-go (s) & $6,7 \pm 1,2$ & $6,7 \pm 1,3$ & $\begin{array}{c}0,88(0,76 \text { to } \\
0,94)\end{array}$ & 0,44 & 6,63 & 1,22 & 18,39 \\
\hline & 6-minute walking $(\mathrm{m})$ & $473,7 \pm 65,7$ & $472 \pm 66,1$ & $\begin{array}{c}0,94(0,87 \text { to } \\
0,97)\end{array}$ & $\begin{array}{c}16,8 \\
1\end{array}$ & 3,55 & $\begin{array}{c}46,5 \\
8\end{array}$ & 9,85 \\
\hline \multicolumn{9}{|l|}{$75-79(n=24)$} \\
\hline & & $\begin{array}{l}\text { Media } 1 \\
( \pm D T)\end{array}$ & $\begin{array}{c}\text { Media } 2 \\
( \pm \mathrm{DT})\end{array}$ & $\mathrm{CCI}(95 \% \mathrm{IC})$ & SEM & $\begin{array}{c}\text { \%SE } \\
M\end{array}$ & SRD & $\begin{array}{c}\text { \%SR } \\
\text { D }\end{array}$ \\
\hline & $\begin{array}{l}\text { Bi-manual handgrip } \\
(\mathrm{Kg})\end{array}$ & $41,1 \pm 9,6$ & $40,4 \pm 9,4$ & $\begin{array}{c}0,94(0,86 \text { to } \\
0,97)\end{array}$ & 2.37 & 5.82 & 6.57 & 16.13 \\
\hline & $\begin{array}{l}\text { Chair Seat-and-reach } \\
(\mathrm{cm})\end{array}$ & $5,3 \pm 7,5$ & $5,3 \pm 7,7$ & $\begin{array}{c}0,85(0,69 \text { to } \\
0,93)\end{array}$ & 2,48 & 33,51 & 6,88 & 92,87 \\
\hline & Functional reach $(\mathrm{cm})$ & $29,1 \pm 7,7$ & $29,4 \pm 6,4$ & $\begin{array}{c}0,88(0,74 \text { to } \\
0,95\end{array}$ & 3,10 & 10,60 & 8,58 & 29,38 \\
\hline
\end{tabular}


Rev.int.med.cienc.act.fís.deporte - vol. 19 - número 76 - ISSN: 1577-0354

\begin{tabular}{|c|c|c|c|c|c|c|c|c|}
\hline \multirow{8}{*}{$\geq 80(n=14)$} & Timed up-and-go (s) & $7,1 \pm 1,0$ & $7,2 \pm 1,0$ & $\begin{array}{c}0,82(0,62 \text { to } \\
0,92)\end{array}$ & 0,64 & 8,94 & 1,77 & 24,79 \\
\hline & 6-minute walking (m) & $455,4 \pm 52,0$ & $460,4 \pm 58,0$ & $\begin{array}{c}0,87(0,71 \text { to } \\
0,94)\end{array}$ & $\begin{array}{c}20,2 \\
0\end{array}$ & 4,41 & $\begin{array}{c}56,0 \\
0\end{array}$ & 12,23 \\
\hline & & $\begin{array}{c}\text { Media } 1 \\
( \pm D T)\end{array}$ & $\begin{array}{c}\text { Media } 2 \\
( \pm D T)\end{array}$ & $\mathrm{CCl}(95 \% \mathrm{IC})$ & SEM & $\begin{array}{c}\% \text { SE } \\
M\end{array}$ & SRD & $\begin{array}{c}\text { \%SR } \\
\text { D }\end{array}$ \\
\hline & $\begin{array}{l}\text { Bi-manual handgrip } \\
(\mathrm{Kg})\end{array}$ & $36,6 \pm 6,3$ & $36,4 \pm 7,4$ & $\begin{array}{c}0,93(0,78 \text { to } \\
0,98)\end{array}$ & 1.82 & 4.98 & 5.04 & 13.81 \\
\hline & $\begin{array}{l}\text { Chair Seat-and-reach } \\
\qquad(\mathrm{cm})\end{array}$ & $1,1 \pm 12,5$ & $0,7 \pm 12,9$ & $\begin{array}{c}0,85(0,60 \text { to } \\
0,95)\end{array}$ & 2,66 & 28,81 & 7,38 & 79,86 \\
\hline & Functional reach $(\mathrm{cm})$ & $24,4 \pm 8,5$ & $24,5 \pm 7,3$ & $\begin{array}{c}0,82(0,52 \text { to } \\
0,94)\end{array}$ & 4,03 & 16,52 & $\begin{array}{c}11,1 \\
8\end{array}$ & 45,78 \\
\hline & Timed up-and-go (s) & $8,1 \pm 1,3$ & $8,2 \pm 1,3$ & $\begin{array}{c}0,92(0,77 \text { to } \\
0,97)\end{array}$ & 0,37 & 4,48 & 1,02 & 12,42 \\
\hline & 6-minute walking $(\mathrm{m})$ & $356,4 \pm 94,4$ & $365 \pm 93,7$ & $\begin{array}{c}0,98(0,93 \text { to } \\
0,99)\end{array}$ & $\begin{array}{c}13,9 \\
5\end{array}$ & 3,87 & $\begin{array}{c}38,6 \\
7\end{array}$ & 10,72 \\
\hline
\end{tabular}

DT: Desviación Típica; CCI: Coeficiente de Correlación Intercalase; IC: 95\% Intervalo de Confianza; SEM: Error Estándar de Medida, SRD: Mínimo Cambio Real

La Tabla 3 muestra la diferencia de medias entre la medición del test y retest para todos los grupos de edad. No se obtuvieron diferencias estadísticamente significativas en función de edad.

Tabla 3. Diferencias entre test y retest en función de grupo de edad $(n=116)$. Análisis ANOVA con

\begin{tabular}{lcccccccc}
\multicolumn{10}{c}{ post hoc de Games-Howel. } \\
\hline \multicolumn{1}{c}{ Prueba } & $\begin{array}{c}\text { Todos } \\
(\mathrm{n}=116)\end{array}$ & $\begin{array}{c}60-64 \\
(\mathrm{n}=21)\end{array}$ & $\begin{array}{c}65-69 \\
(\mathrm{n}=27)\end{array}$ & $\begin{array}{c}70-74 \\
(\mathrm{n}=30)\end{array}$ & $\begin{array}{c}75-79 \\
(\mathrm{n}=24)\end{array}$ & $\begin{array}{c}\geq 80 \\
(\mathrm{n}=14)\end{array}$ & $\mathrm{F}$ & $\mathrm{P}$ \\
\hline $\begin{array}{l}\text { Dinamometría bi- } \\
\text { manual (Kg) }\end{array}$ & $0,2 \pm 0,3$ & $0,0 \pm 0,7$ & $0,3 \pm 3,2$ & $0,8 \pm 0,7$ & $-0,8 \pm-0,2$ & $-0,2 \pm 1,1$ &, 479 &, 751 \\
$\begin{array}{l}\text { Chair Seat-and- } \\
\text { reach (cm) }\end{array}$ & $0,0 \pm-0,1$ & $-0,8 \pm 1,3$ & $1,5 \pm-2,2$ & $-0,6 \pm 0,5$ & $0,0 \pm 0,2$ & $-0,4 \pm 0,4$ & 1,167 &, 330 \\
$\begin{array}{l}\text { Functional reach } \\
\text { (cm) }\end{array}$ & $0,8 \pm-0,4$ & $0,6 \pm 0,5$ & $1,4 \pm 0,5$ & $1,0 \pm-0,6$ & $0,3 \pm-1,4$ & $0,1 \pm-1,1$ &, 303 &, 875 \\
$\begin{array}{l}\text { Timed up-and-go } \\
\text { (s) }\end{array}$ & $0,0 \pm 0,0$ & $0,0 \pm-0,2$ & $-0,1 \pm-0,1$ & $0,0 \pm 0,1$ & $0,0 \pm 0,0$ & $0,1 \pm 0,1$ &, 219 &, 928 \\
$\begin{array}{l}6-\text { minute } \\
\text { caminando (m) }\end{array}$ & $1,7 \pm-2,0$ & $3,3 \pm 2,6$ & $-2,4 \pm-8,9$ & $-1,7 \pm 0,3$ & $5,0 \pm 6,0$ & $8,6 \pm-0,7$ &, 688 &, 602 \\
\hline
\end{tabular}

Valores como media \pm desviación típica

No hubo diferencias significativas entre los grupos que usaron la prueba post hoc de Games-Howell.

La Tabla 4 muestra el ES de las diferencias test-retest entre los grupos de edad. Todos los ES fueron bajos, excepto la prueba time up-and-go en la que fue moderada. 
Rev.int.med.cienc.act.fís.deporte - vol. 19 - número 76 - ISSN: 1577-0354

\begin{tabular}{|c|c|c|c|c|c|}
\hline & $\begin{array}{l}\text { Grupo de } \\
\text { edad }\end{array}$ & $\begin{array}{l}65-69 \\
(\mathrm{n}=27) \\
\end{array}$ & $70-74(n=30)$ & $75-79(n=24)$ & $\geq 80(n=14)$ \\
\hline \multirow[t]{4}{*}{$\begin{array}{l}\text { Dinamometria bi-manual } \\
(\mathrm{Kg})\end{array}$} & $\begin{array}{l}60-64 \\
(\mathrm{n}=21)\end{array}$ & 0,2 & 1,6 & $-3,2$ & $-0,3$ \\
\hline & $\begin{array}{l}65-69 \\
(n=27)\end{array}$ & & 0,3 & $-0,6$ & $-0,2$ \\
\hline & $\begin{array}{c}70-74 \\
(\mathrm{n}=30)\end{array}$ & & & $-3,6$ & $-1,1$ \\
\hline & $\begin{array}{l}75-79 \\
(n=24)\end{array}$ & & & & 0,9 \\
\hline \multirow[t]{5}{*}{$\begin{array}{l}\text { Chair Seat-and-reach } \\
\text { (cm) }\end{array}$} & $\begin{array}{l}60-64 \\
(n=21)\end{array}$ & 1,3 & 0,2 & 1,1 & 0,5 \\
\hline & $\begin{array}{l}65-69 \\
(n=27)\end{array}$ & & $-1,6$ & $-1,3$ & $-1,5$ \\
\hline & $\begin{array}{l}70-74 \\
(n=30)\end{array}$ & & & 1,7 & 0,4 \\
\hline & $\begin{array}{l}75-79 \\
(n=24)\end{array}$ & & & & $-1,3$ \\
\hline & $\begin{array}{l}60-64 \\
(\mathrm{n}=27)\end{array}$ & $-0,2$ & 0,9 & 0,2 & 1,2 \\
\hline \multirow[t]{4}{*}{ Functional reach $(\mathrm{cm})$} & $\begin{array}{l}60-64 \\
(n=21)\end{array}$ & 0,3 & 0,1 & $-0,1$ & $-0,2$ \\
\hline & $\begin{array}{l}65-69 \\
(n=27)\end{array}$ & & $-0,7$ & $-1,2$ & $-1,6$ \\
\hline & $\begin{array}{l}70-74 \\
(n=30)\end{array}$ & & & $-0,7$ & $-1,1$ \\
\hline & $\begin{array}{l}75-79 \\
(n=24)\end{array}$ & & & & $-0,2$ \\
\hline \multirow[t]{4}{*}{ Timed up-and-go (s) } & $\begin{array}{l}60-64 \\
(n=21)\end{array}$ & $-0,7$ & 0,0 & 0,0 & 0,7 \\
\hline & $\begin{array}{l}65-69 \\
(n=27)\end{array}$ & & 1,0 & 2,0 & 2,0 \\
\hline & $\begin{array}{l}70-74 \\
(n=30)\end{array}$ & & & 0,0 & 1,0 \\
\hline & $\begin{array}{l}75-79 \\
(n=24)\end{array}$ & & & & 2,0 \\
\hline \multirow[t]{4}{*}{ 6-minutos caminando $(\mathrm{m})$} & $\begin{array}{l}60-64 \\
(n=21)\end{array}$ & $-1,0$ & $-2,8$ & 0,4 & 3,2 \\
\hline & $\begin{array}{l}65-69 \\
(n=27)\end{array}$ & & 0,4 & 1,0 & 2,3 \\
\hline & $\begin{array}{l}70-74 \\
(n=30)\end{array}$ & & & 1,8 & 18,6 \\
\hline & $\begin{array}{l}75-79 \\
(n=24)\end{array}$ & & & & 0,1 \\
\hline
\end{tabular}

\section{DISCUSIÓN}

En general, la fiabilidad de las pruebas en los diferentes grupos de edad fue buena y el análisis de ANOVA mostró que el envejecimiento no afecta los valores del test y el retest.

La condición física disminuye gradualmente acorde a la edad, acelerando progresivamente después de los 60 años (Milanović et al., 2013). Muchos estudios entregan valores normativos de la condición física y como estos valores 
van descendiendo según avanza la edad (Rikli \& Jones, 2013; Sardinha et al., 2015; Vagetti et al., 2015), sin embargo hay pocos precedentes de investigaciones analizando la fiabilidad de los instrumentos de condición física en personas mayores (Dewhurst \& Bampouras, 2014; Steffen, Hacker, \& Mollinger, 2002). Además, ninguno de ellos analiza si los valores de fiabilidad de estas pruebas se ven modificados en función de la edad de las personas evaluadas. Los resultados de este estudio muestran que no hay diferencias significativas en función de la edad en el error de medida calculado mediante test-retest.

En relación a la aplicación de test y retest, estudios previos han analizado la fiabilidad de varias pruebas de condición física realizadas en diferentes momentos de un mismo día. Este estudio muestra la que la prueba de velocidad máxima de marcha de 6 minutos caminando, prueba de chair sit and reach y fuctional reach poseen una excelente fiabilidad. Dado que el estudio actual evalúa la fiabilidad entre sesiones con una semana de separación, parece razonable que la fiabilidad informada en este estudio previo sea más alta que la obtenida en este.

Para el cálculo de la fiabilidad se utilizó el valor del ICC al ser un índice ampliamente utilizado. No obstante, la interpretación puede causar problemas al existir diversa literatura con usos de umbrales distintos. En este caso, se utilizó la clasificación propuesta por Munro y cols. (Munro, Visintainer, \& Page, 1986) que considera como fiabilidad moderada valores comprendidos entre 0,50 y0,69, alta los valores comprendidos entre 0,70 y 0,89 , y excelente valores superiores a 0,90 .

Siguiendo esta clasificación, los resultados obtenidos muestran que la fiabilidad para todos los grupos de edad evaluados fue alta en las pruebas sit-and-reach, functional reach test y timed up-and-go, mientras que la obtenida en dinamometría bi-manual y 6 minutos caminando fue excelente. Con estos resultados se puede indicar que todas las pruebas evaluadas poseen una alta fiabilidad en todos los grupos de edad. Al analizar las diferencias en función de la edad, el test de fuctional reach es la prueba con más cambios en su fiabilidad entre los grupos analizados (ICC 0,71-0,88). El análisis de ANOVA no muestra diferencias estadísticamente significativas en función de la edad en ninguna de las pruebas evaluadas. En cuanto a las diferencias según el tamaño del efecto entre los distintos grupos de edad, todas las pruebas tienen un tamaño bajo exceptuando el test de time up-and-go que tiene un tamaño moderado. Este resultado indica que no hay cambios relevantes por grupo de edad en cuanto a la magnitud de la diferencia de medias obtenidas entre el test y el retest.

Los estudios como este, enfocados a analizar a fiabilidad de instrumentos de evaluación, ayudan a los profesionales que usan estas pruebas en su quehacer diario. Concretamente, conocer los datos de fiabilidad mostrados en este estudio ayuda a conocer la precisión en la evaluación de pruebas que pueden ser utilizadas para clasificar el riesgo de padecer baja funcionalidad física en adulto mayor (Merellano-Navarro et al., 2017; Rikli \& Jones, 2013; Sardinha et al., 
2015). También es útil para conocer la diferencia mínima necesaria que se debe alcanzar en terapias enfocadas a la mejora de la condición física de esta población para asegurarse que el efecto encontrado es mayor que el error que se debe asumir durante la medición. Adicionalmente, en entornos de investigación, un uso importante de los parámetros de fiabilidad de las pruebas a utilizar es la estimación del tamaño de la muestra en estudios experimentales (Hopkins, 2000).

\section{LIMITACIONES}

La muestra de este estudio no considera hombres mayores. Esto hace que los resultados no se puedan generalizar a esta población. Otra limitación a tener en cuenta es que los participantes de los grupos de mayor edad son poco numerosos, así como que todos los participantes son personas mayores autovalentes que viven en la comunidad por lo que los resultados no son representativos de aquellas personas con problemas de capacidad funcional.

\section{CONCLUSIONES}

Las pruebas de condición física analizadas en este estudio muestran buena fiabilidad en mujeres mayores. A través del análisis test-retest por grupos de edad se demostró que la fiabilidad en esta población no se ve afectada por el incremento de la edad en esta población.

\section{REFERENCIAS BIBLIOGRÁFICAS}

Chang, Y., Wang, J., Chen, H., \& Hu, S. (2017). Aerobic-synergized exercises may improve fall-related physical fitness in older adults. The Journal of sports medicine and physical fitness, 57(5), 660-669. https://doi.org/10.23736/s0022-4707.16.05728-5

Chen, H., Lin, C., \& Yu, L. (2009). Normative physical fitness scores for community-dwelling older adults. $J$ Nurs Res, 17(1), 30-41. https://doi.org/10.1097/JNR.0b013e3181999d4c

Chen, T., Tseng, W., Huang, G., Chen, H., Tseng, K., \& Nosaka, K. (2017). Superior Effects of Eccentric to Concentric Knee Extensor Resistance Training on Physical Fitness, Insulin Sensitivity and Lipid Profiles of Elderly Men. Frontiers in physiology, 8, 209. https://doi.org/10.3389/fphys.2017.00209

Chung, P., Zhao, Y., Liu, J., \& Quach, B. (2017). A canonical correlation analysis on the relationship between functional fitness and health-related quality of life in older adults. Archives of gerontology and geriatrics, 68, 44-48. https://doi.org/10.1016/j.archger.2016.08.007

Deurenberg, P., Andreoli, A., Borg, P., Kukkonen-Harjula, K., de Lorenzo, A., van Marken Lichtenbelt, W. D., . . Vollaard, N. (2001). The validity of predicted body fat percentage from body mass index and from impedance in samples of five European populations. Eur J Clin Nutr, 55(11), 973-979. https://doi.org/10.1038/sj.ejcn.1601254 
Dewhurst, S., \& Bampouras, T. (2014). Intraday reliability and sensitivity of four functional ability tests in older women. American journal of physical medicine \& rehabilitation, 93(8), 703-707. https://doi.org/10.1097/PHM.0000000000000078

Duncan, P., Weiner, D., Chandler, J., \& Studenski, S. (1990). Functional reach: a new clinical measure of balance. Journal of gerontology, 45(6), M192197. https://doi.org/10.1093/geronj/45.6.M192

Gusi, N., Hernandez-Mocholi, M. A., \& Olivares, P. R. (2015). Changes in HRQoL after 12 months of exercise linked to primary care are associated with fitness effects in older adults. European journal of public health, 25(5), 873-879. https://doi.org/10.1093/eurpub/ckv079

Hopkins, W. G. (2000). Measures of reliability in sports medicine and science. Sports Med, 30(1), 1-15. https://doi.org/10.2165/00007256-200030010$\underline{00001}$

Jones, C. J., Rikli, R. E., Max, J., \& Noffal, G. (1998). The reliability and validity of a chair sit-and-reach test as a measure of hamstring flexibility in older

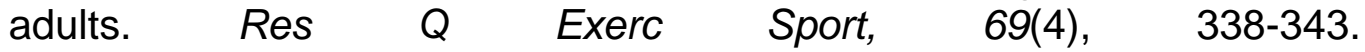
https://doi.org/10.1080/02701367.1998.10607708

Krause, M. P., Januário, R. S. B., Hallage, T., Haile, L., Miculis, C. P., Gama, M. P. R., ... da Silva, S. G. (2009). A comparison of functional fitness of older Brazilian and American women. $J$ Aging Phys Act, 17(387-397). https://doi.org/10.1123/japa.17.4.387

Merellano-Navarro, E., Collado-Mateo, D., García-Rubio, J., Gusi, N., \& Olivares, P. R. (2017). Criterion-Referenced Fitness Standards associated with maintaining functional capacity in Chilean Older Adults. Rejuvenation Research. https://doi.org/10.1089/rej.2016.1913

Milanović, Z., Pantelić, S., Trajković, N., Sporiš, G., Kostić, R., \& James, N. (2013). Age-related decrease in physical activity and functional fitness among elderly men and women. Clinical interventions in aging, 8, 549. https://doi.org/10.2147/CIA.S44112

Munro, B. H., Visintainer, M. A., \& Page, E. B. (1986). Statistical methods for health care research. Philadelphia: JB Lippincott.

Oja, P. (1995). Eurofit for Adults: Assessment of Health-Related Fitness. Tampere, Finland: Council of Europe Publishing.

Olivares, P., Gusi, N., Prieto, J., \& Hernandez-Mocholi, M. (2011). Fitness and health-related quality of life dimensions in community-dwelling middle aged and older adults. Health and quality of life outcomes, 9, 117. https://doi.org/10.1186/1477-7525-9-117

Podsiadlo, D., Richardson, S. (1991). The timed "up \& go": A test of basic functional mobility for frail elderly persons. Journal of the American Geriatrics Society, 39, 142-148. https://doi.org/10.1111/i.15325415.1991.tb01616.x

Rikli, R., \& Jones, C. (1999a). Development and validation of a functional fitness test for community-residing older adults. J Aging Phys Act, 7(2), 129-161. https://doi.org/10.1123/japa.7.2.129

Rikli, R., \& Jones, C. (1999b). Functional fitness normative scores for communityresiding older adutls, ages 60-94. J Aging Phys Act, 7(3), 60-179. https://doi.org/10.1123/japa.7.2.162 
Rikli, R., \& Jones, C. (2001). Senior Fitness Test Manual Champaign, IL.

Rikli, R., \& Jones, C. (2013). Development and validation of criterion-referenced clinically relevant fitness standards for maintaining physical independence in later years. The Gerontologist, 53(2), 255-267. https://doi.org/10.1093/geront/gns071

Rodríguez, F. A., Gusi, N., Valenzuela, A., Nácher, S., Nogués, J., \& Marina, M. (1998). [Evaluation of health-related fitness in adults (I): background and protocols of the AFISAL-INEFC battery]. Apunts. Educ. Fís. Deportes, 52, 54-75.

Rodriguez, F. A., Valenzuela, A., Gusi, N., Nacher, S., \& Gallardo, I. (1998). [Evaluation of health-related fitness in adults (II): reliability, applicability and normative values of the AFISAL-INEFC battery]. Apunts. Educ. Fís. Deportes, 54, 54-65.

Sardinha, L., Santos, D., Marques, E., \& Mota, J. (2015). Criterion-referenced fitness standards for predicting physical independence into later life. Experimental gerontology, $\quad 61, \quad 142-146$. https://doi.org/10.1016/i.exger.2014.12.012

Shaulis, D., Golding, L., \& Tandy, R. (1994). Reliability of the AAHPERD functional fitness assessment across multiple practice sessions in older men an women. J Aging Phys Act, 2(3), 273-279. https://doi.org/10.1123/japa.2.3.273

Shrout, P., \& Fleiss, J. (1979). Intraclass correlations: uses in assessing rater reliability. Psychological bulletin, 86(2), 420-428. https://doi.org/10.1037/0033-2909.86.2.420

Steffen, T. M., Hacker, T. A., \& Mollinger, L. (2002). Age-and gender-related test performance in community-dwelling elderly people: Six-Minute Walk Test, Berg Balance Scale, Timed Up \& Go Test, and gait speeds. Physical therapy, 82(2), 128-137. https://doi.org/10.1093/ptj/82.2.128

Thomas, S., Reading, J., \& Shephard, R. J. (1992). Revision of the Physical Activity Readiness Questionnaire (PAR-Q). Can J Sport Sci, 17(4), 338345.

Tomas, J., \& Nelson, J. (2005). Research Methods in Physical Activity (5 ed.). Champaign, IL: Human Kinetics.

Vagetti, G. C., Barbosa Filho, V. C., de Oliveira, V., Mazzardo, O., Moreira, N. B., Gomes, A. C., \& de Campos, W. (2015). Functional fitness in older women from southern brazil: normative scores and comparison with different countries. Brazilian Journal of Kinanthropometry and Human Performance, 17(4), 472-484. $\quad$ https://doi.org/10.5007/1980$\underline{0037.2015 \mathrm{v} 17 \mathrm{n} 4 \mathrm{p} 472}$

Weir, J. (2005). Quantifying test-retest reliability using the intraclass correlation coefficient and the SEM. Journal of strength and conditioning research, 19(1), 231-240. https://doi.org/10.1519/15184.1

\section{Número de citas totales / Total references: $31(100 \%)$ Número de citas propias de la revista / Journal's own references: $0(0 \%)$}

